\title{
Expression of Cyclin D1 in normal and hyperplastic endometrium
}

\author{
Parveen R. Kundu, Anjali Sindhu*, Swaran Kaur, Amrita Kulhria, Ritika Hooda
}

Department of Pathology, Bhagat Phool Singh Government Medical College for Women, Khanpur Kalan, Sonepat, Haryana, India

Received: 18 August 2019

Accepted: 30 September 2019

*Correspondence:

Dr. Anjali Sindhu,

E-mail: sindhuanjali.01@gmail.com

Copyright: (C) the author(s), publisher and licensee Medip Academy. This is an open-access article distributed under the terms of the Creative Commons Attribution Non-Commercial License, which permits unrestricted non-commercial use, distribution, and reproduction in any medium, provided the original work is properly cited.

\section{ABSTRACT}

Background: Endometrial hyperplasia is characterised by increased gland to stroma ratio with varying degree of atypia. Cyclin D1 is a protein playing important role during the $\mathrm{G} 1 \rightarrow \mathrm{S}$ phase transition in the cell cycle. The present study evaluated the expression of Cyclin D1 in normal and hyperplastic endometrium.

Methods: A cross sectional study was conducted over a period of 1 year. We evaluated and compared the expression of Cyclin D1 in 56 endometrial samples including 24 cases of simple hyperplasia, 12 cases of complex hyperplasia and 10 cases each of secretory and proliferative endometrium.

Results: A substantial increase in expression of Cyclin D1 was seen in hyperplastic endometrium compared to normal endometrium. Moreover, complex hyperplasia showed the maximum positivity for Cyclin D1.

Conclusions: Cyclin D1 may play a stimulatory role in the proliferation of endometrial glands and hence may be involved in endometrial tumorigenesis.

Keywords: Cyclin D1, Endometrium, Hyperplasia

\section{INTRODUCTION}

The endometrium is the innermost layer of the uterine cavity surrounded by myometrium and serosa. It has great proliferative and regenerative potential. The ovarian and pituitary hormones lead to changes in the endometrium resulting in two-phases of endometrium namely proliferative and secretory. Endometrial hyperplasia is characterized by the proliferation of endometrial glands resulting in a greater than normal gland-to-stroma ratio which leads to varying degrees of architectural complexity and cytologic atypia. It is clinically significant as it can progress to endometrial adenocarcinoma. ${ }^{1}$

The most commonly used classification system for endometrial hyperplasia is the World Health Organization (WHO) 1994 classification system which is based on architectural disruption and cytological atypia. It classifies endometrial hyperplasia into four types namely simple hyperplasia without atypia, simple hyperplasia with atypia, complex hyperplasia without atypia and complex hyperplasia with atypia. Cytological atypia increases the risk for both, progression to endometrial carcinoma and coexistent endometrial carcinoma in women with endometrial hyperplasia. ${ }^{2}$ The new WHO classification of endometrial hyperplasia 2014 categorizes endometrial hyperplasia into two namely hyperplasia without atypia and atypical hyperplasia/endometrioid intraepithelial neoplasia. ${ }^{3}$

The risk of progression of endometrial hyperplasia to endometrial carcinoma varies from $1 \%$ to $29 \%$ with the minimum $1 \%$ being in simple hyperplasia without atypia and maximum of $29 \%$ in complex hyperplasia with atypia. ${ }^{4}$ Various parameters like stromal invasion, increased degrees of nuclear atypia, mitotic activity, cellular stratification, and epithelial necrosis are associated with a greater likelihood of endometrial carcinoma. ${ }^{5}$ 
CyclinD1 is a protein encoded by the CCND1 gene located on chromosome $11 \mathrm{q} 13 .{ }^{6}$ It plays a crucial role in the regulation of cell cycle by acting at the key ratelimiting point i.e. $\mathrm{G} 1 \rightarrow \mathrm{S}$ phase transition. It can activate CDK4 or CDK6 to phosphorylate a series of key substrates, such as protein $\mathrm{RB}$, to promote synthesis of DNA and accelerate the cells proliferation. It thus promotes cell cycle progression from $\mathrm{G} 1$ to $\mathrm{S}$ phase.

Mutations, amplification and over-expression of CCND1 gene can alter cell cycle progression and may contribute to tumorigenesis. These have been observed frequently in a variety of tumors like multiple myeloma, $\mathrm{T}$ cutaneous lymphomas and solid cancers e.g. oral squamous cell carcinoma, lung cancer, melanoma, breast cancer and hepatocellular carcinoma. ${ }^{7-10}$ Proliferative endometrial glands and stroma, even when actively mitotic do not over express Cyclin D1. Studies in the past have observed the over expression of Cyclin D1 in endometrial hyperplasia and carcinoma. In the present study we evaluated the pattern of cyclin D1 expression in normal and hyperplastic endometrium.

\section{METHODS}

The present study was conducted over a period of one year in the Department of Pathology at Bhagat Phool Singh Government Medical College for Women, Khanpur Kalan, Sonepat. A total of 56 cases including 24 cases of simple hyperplasia and 12 cases of complex hyperplasia and 10 cases each of secretory and proliferative endometrium were considered in the study.

Cases included were ranging in age from 27 years to 78 years with the maximum number of cases were in the age group of 41-50 years. The study material comprised of hysterectomy and endometrial biopsies/curettings specimen. Tissues were routinely fixed in buffered formalin and embedded in paraffin wax followed by routine haematoxylin and eosin staining. Standard procedure for Immunohistochemistry for Cyclin D1 using flex monoclonal rabbit human Cyclin Clone EP 12 was performed.

\section{Interpretation of immunohistochemistry}

Cyclin D1 staining was evaluated in terms of two parameters namely the intensity of nuclear staining and the extent (percentage of positive cells). The intensity of nuclear staining was graded as no staining (0), weak (1+), moderate $(2+)$, or strong $(3+)$. The extent was semiquantitatively estimated with a range of $0 \%$ to $100 \%$. Percentage was estimated by counting at least 50 nuclei and then calculating the ratio of immune-reactive nuclei to total number of nuclei multiplied by 100 ; percentages were rounded to the nearest $10 \%$. When less than $10 \%$ of cells were positive, a score of 0 was used, $11 \%$ to $30 \%$ cell positivity was scored as $1+, 31 \%$ to $60 \%$ positivity was scored as $2+$, and more than $60 \%$ positive cells were labelled as $3+$ grade.

\section{Statistical analysis}

Data was analysed using the statistical package SPSS version 22. Chi-square was used to analyse the data and $P$ value was calculated wherever required. $P$ value of 0.05 or less was considered as statistically significant.

\section{RESULTS}

\section{Distribution of cases}

Out of total cases, 24 cases were of simple hyperplasia along with 12 cases of complex hyperplasia and 10 cases each of secretory and proliferative endometrium. Out of 12 cases of complex hyperplasia, five cases were complex hyperplasia without atypia and seven cases were complex hyperplasia with atypia.

\section{Demographics}

Cases included were ranging in age from 27 years to 78 years with the maximum number of cases in the age group of 41-50 years. The mean age for simple hyperplasia was 43.9 years with maximum number of cases in the age group of 41-50 years. For complex hyperplasia, the mean age of 52.3 years was calculated while maximum number of cases fall in the age group of 41-50 years.

\section{Clinical presentation}

The most common presenting feature was vaginal bleeding which was found in 23 cases of simple hyperplasia and 12 cases of complex hyperplasia. One case of simple hyperplasia presented with uterovaginal prolapse.

\section{Cyclin D1 immunostaining}

Cases with weak staining and less than $10 \%$ extent were considered to be negative. Out of total fifty-six cases, thirty-two cases $(57.14 \%)$ showed Cyclin D1 positivity. These included sixteen cases $(66.7 \%)$ of simple hyperplasia, eleven cases $(91.6 \%)$ of complex hyperplasia, three cases $(30 \%)$ of secretory and two cases $(20 \%)$ of proliferative phase endometrium (Table 1$)$.

In secretory phase endometrium, $70 \%$ cases had an extent of zero. Similarly, in proliferative phase endometrium, extent of staining was graded zero in $80 \%$ cases. Out of twenty-four cases of simple hyperplasia of endometrium, extent of staining was graded $2+$ in ten cases $(41.7 \%)$.

In case of complex hyperplasia of endometrium, $50 \%$ cases showed an extent of $3+$, followed by four cases $(33.3 \%)$ of $2+$ extent and one case $(8.3 \%)$ of an extent of $1+$. Only single case $(8.3 \%)$ was found with zero extent (Table 2). 
Table 1: Percentage positivity of Cyclin D1 in normal and hyperplastic endometrium.

\begin{tabular}{|llll|}
\hline Diagnosis & No. of cases (n) & Cyclin D1 positive & Percentage positivity (\%) \\
\hline Proliferative phase & 10 & 02 & 20 \\
\hline Secretory phase & 10 & 03 & 30 \\
\hline Simple hyperplasia & 24 & 16 & 66.7 \\
\hline Complex hyperplasia & 12 & 11 & 91.6 \\
\hline Total & $\mathbf{5 6}$ & $\mathbf{3 2}$ & $\mathbf{5 7 . 1 4}$ \\
\hline
\end{tabular}

Table 2: Extent of Cyclin D1 expression in normal and hyperplastic endometrium.

\begin{tabular}{|lllll|}
\hline Diagnosis & 0 & $1+$ & $2+$ & $3+$ \\
\hline Secretory endometrium & 07 & 02 & 01 & 00 \\
\hline Proliferative endometrium & 08 & 02 & 00 & 00 \\
\hline Simple hyperplasia endometrium & 08 & 03 & 10 & 03 \\
\hline Complex hyperplasia endometrium & 01 & 01 & 04 & 06 \\
\hline
\end{tabular}

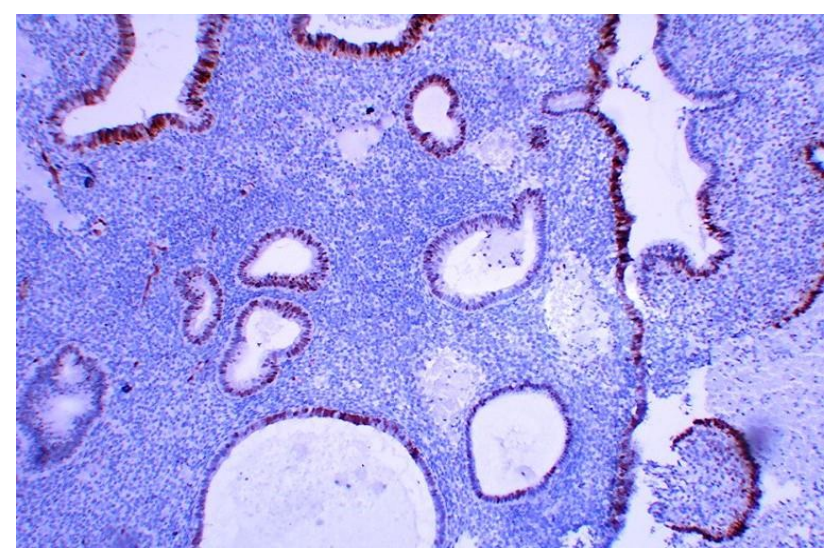

Figure 1: Simple hyperplasia endometrium: Cyclin D1 expression show extent 2+ (IHC 100X).

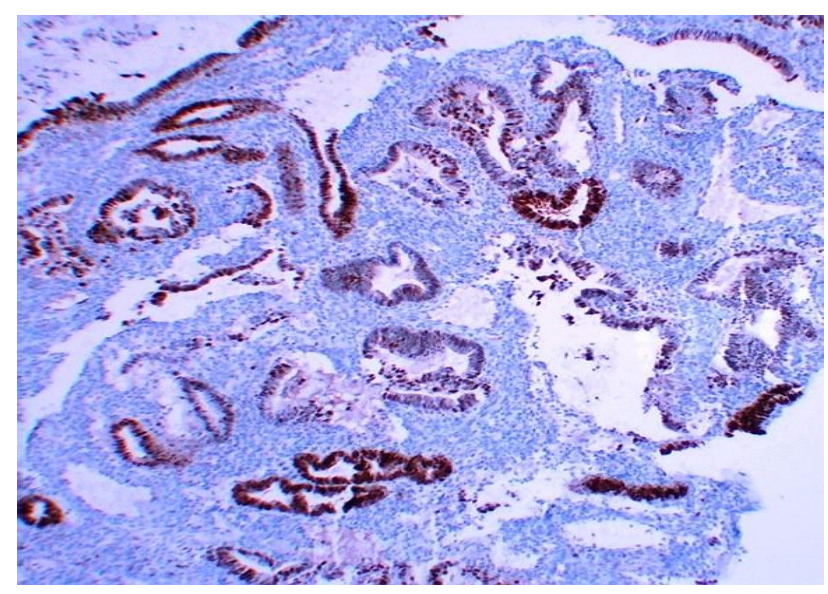

Figure 2: Complex hyperplasia: Cyclin D1 expression showing extent 3+ (IHC 40X)

Majority of cases of simple hyperplasia had an extent of $2+$ (Figure 1) while most of the cases of complex hyperplasia showed an extent of $3+$ (Figure 2). On comparing the extent of Cyclin D1 staining between non neoplastic and hyperplastic endometrium, significant difference was found between secretory endometrium and simple hyperplasia $(\mathrm{p}=0.049)$ and secretory endometrium and complex hyperplasia $(\mathrm{p}=0.0027)$. Also, significant difference was found between proliferative endometrium and simple hyperplasia $(\mathrm{p}=0.0129)$ and proliferative endometrium and complex hyperplasia $(p=0.00066)$. However, no significant difference in extent was found between secretory endometrium and proliferative endometrium $(\mathrm{p}=0.606)$ and simple and complex hyperplasia $(\mathrm{p}=0.102)$.

Table 3: Intensity of Cyclin D1 expression in normal and hyperplastic endometrium.

\begin{tabular}{|lllll|}
\hline Diagnosis & 0 & $1+$ & $2+$ & $3+$ \\
\hline Secretory endometrium & 07 & 02 & 01 & 00 \\
\hline Proliferative endometrium & 07 & 02 & 01 & 00 \\
\hline Simple hyperplasia endometrium & 07 & 10 & 05 & 02 \\
\hline Complex hyperplasia endometrium & 01 & 04 & 06 & 01 \\
\hline
\end{tabular}

In terms of intensity, seven cases $(70 \%)$ each of both secretory and proliferative endometrium showed an intensity of grade zero. Intensity of staining was found to be of grade $1+$ in ten cases $(41.7 \%)$ of simple hyperplasia. In complex hyperplasia, $50 \%$ cases showed an extent of $3+$ (Table 3$)$.

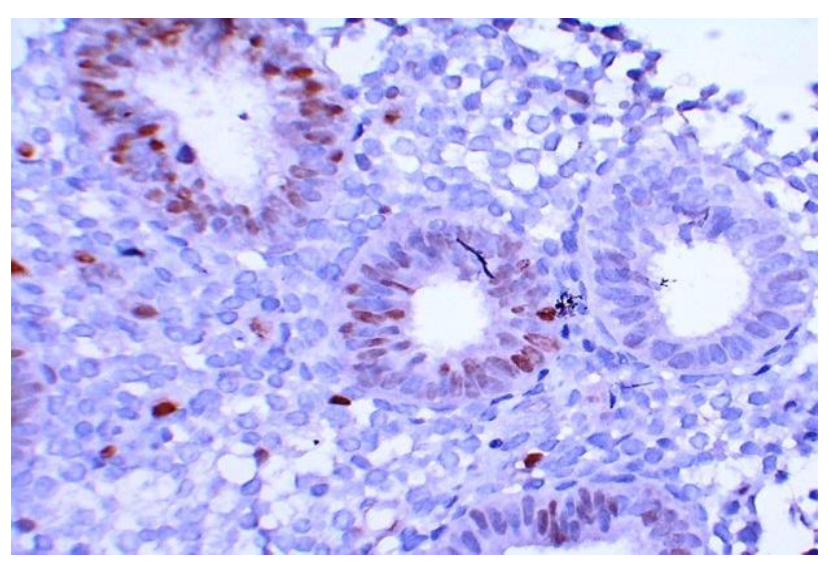

Figure 3: Simple hyperplasia endometrium: Cyclin D1 expression show intensity 1+ (IHC 200X).

In case of simple hyperplasia, maximum cases showed $1+$ intensity of Cyclin D1 staining (Figure 3). Majority of 
cases of complex hyperplasia had a $2+$ intensity and one case showed 3+ intensity (Figure 4, 5). On comparing the intensity of Cyclin D1 expression, significant difference was seen between secretory endometrium and simple hyperplasia $(\mathrm{p}=0.027)$ and secretory endometrium and complex hyperplasia $(\mathrm{p}=0.0027)$. Also, significant difference in intensity of Cyclin D1 expression was seen between proliferative endometrium and simple hyperplasia $(\mathrm{p}=0.027)$ and proliferative endometrium and complex hyperplasia $(\mathrm{p}=0.0027)$. However, no significant difference in intensity of Cyclin D1 expression was found between secretory endometrium and proliferative endometrium $(\mathrm{p}=1)$ and simple hyperplasia and complex hyperplasia $(\mathrm{p}=0.156)$.

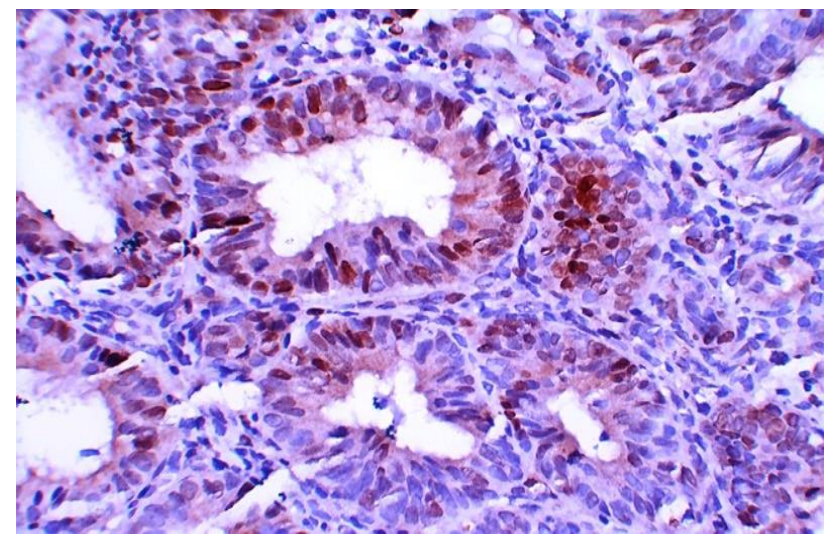

Figure 4: Complex hyperplasia: Cyclin D1 expression showing intensity 2+ (IHC 200X).

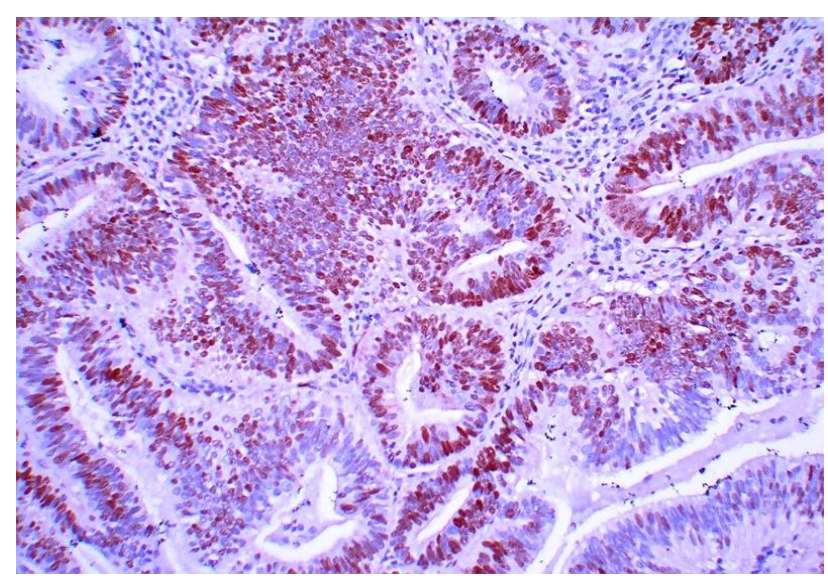

Figure 5: Complex hyperplasia: Cyclin D1 expression showing intensity 3+ (IHC 200x).

\section{DISCUSSION}

The innermost lining of the uterine cavity is endometrium which is surrounded by myometrium and a serosal covering. It undergoes cyclical morphological changes under the effect of ovarian and pituitary hormones.

Prolonged estrogen stimulation that is unopposed by progesterone often results in proliferation of endometrial glands leading to greater than normal gland-to-stroma ratio which results in varying degrees of architectural complexity and cytologic atypia, a lesion designated histopathologically as endometrial hyperplasia. It has the potential to transform into endometrial malignancy.

The exact mechanism of estrogen's role in the transformation of normal endometrium to hyperplasia and cancer is unknown. Various genetic alterations have been found to be associated with hyperplasia namely microsatellite instability and defects in DNA mismatch repair genes (MLH1, MSH2, MSH6, PMS2) associated with the Lynch syndrome. ${ }^{11}$ PTEN(Phosphatase and tensin homologue) tumor suppressor gene mutations have also been found in $55 \%$ of hyperplasia cases and $83 \%$ of hyperplasia cases once it has progressed to endometrial cancer. $^{12}$

Many studies in the past have demonstrated the role of cyclin D1 in endometrial carcinogenesis.

Quddus MR et al, analysed the extent of Cyclin D1 positivity in secretory endometrium and found that out of total 11 cases, 5 were negative and 6 were grade $1+$ positive. ${ }^{13}$ Also, out of 13 cases of proliferative endometrium, 8 were negative and 5 had $1+$ extent of staining.

The findings of our study bear close resemblance to the study by Suri et al, in which four out of the ten cases $(40 \%)$ of secretory endometrium were positive amongst which, two cases (20\%) showed an extent of $1+$ and remaining two cases $(20 \%)$ showed an extent of $2+.{ }^{14}$ In the same study, three out of the ten cases $(30 \%)$ of proliferative endometrium showed Cyclin D1 positivity with an extent of grade 1+ and the remaining seven cases $(70 \%)$ were negative.

The positivity of Cyclin D1 in case of simple hyperplasia has been reported as zero percent in the studies conducted by Tsuda et al, Chaudhary et al, and Kala et al. ${ }^{15-17}$

In the study by Nishimura et al, $22.2 \%$ positivity for Cyclin D1 in cases of simple hyperplasia was noted while Suri et al observed $50 \%$ positivity in cases of simple hyperplasia. ${ }^{14,18}$ Quddus et al, reported $57 \%$ positivity in simple hyperplasia. ${ }^{13}$ In the present study, we found out that 16 out of total twenty-four cases $(66.7 \%)$ of simple hyperplasia were positive for Cyclin D1.

Cyclin D1 positivity in complex hyperplasia has been reported as zero percent by Tsuda et al, Nishimura et al, reported $27.2 \%$ positivity while Chaudhary et al, reported $33 \%$ positivity and Liang et al, reported $49 \%$ positivity for Cyclin D1 in case of complex hyperplasia. ${ }^{6,15,16,18}$ Suri et al and Quddus et al reported $63.6 \%$ and $71 \%$ positivity respectively in cases of complex hyperplasia. ${ }^{13,14}$

In the present study, complex hyperplasia showed $91.6 \%$ (11/12) positivity for Cyclin D1 resembling the study by 
Kala et al, who observed $100 \%$ positivity for Cyclin D1 in cases of complex hyperplasia. ${ }^{17}$

In our study, the expression of Cyclin D1 increased greatly from secretory and proliferative endometrium to endometrial hyperplasia. Also, Cyclin D1 is maximally expressed in cases of complex hyperplasia. Thus, our study suggests the hypothesis that increasing cyclin D1 expression may be associated with proliferation of endometrial glands resulting in endometrial hyperplasia.

\section{CONCLUSION}

There was a statistically significant difference in expression of cyclin D1 both in terms of extent as well as intensity of staining between normal secretory and proliferative phase endometrium versus endometrial hyperplasia. The expression of cyclin D1 increased substantially from secretory and proliferative endometrium to endometrial hyperplasia. This indicates that cyclin D1 may play a stimulatory role in the growth of endometrial glands where it is associated with tumorigenesis.

Funding: No funding sources Conflict of interest: None declared

Ethical approval: Not required

\section{REFERENCES}

1. Chiang WJ. Premalignant lesions of the endometrium. Medscape. 2016:1-9.

2. Salman MC, Usubutun A, Boynukalin K, Yuce K. Comparison of WHO and endometrial intraepithelial neoplasia classifications in predicting the presence of coexistent malignancy in endometrial hyperplasia. J Gynecol Oncol. 2010;21(2):97-101.

3. Emons G, Beckmann MW, Schmidt D, Mallmann P. New WHO classification of endometrial hyperplasias. 2015; 75(2):135-6.

4. Kurman RJ, Kaminski PF, Norris HJ. The behavior of endometrial hyperplasia. A long-term study of "untreated" hyperplasia in 170 patients. Cancer. 1985;56(2):403-12.

5. Kurman RJ, Norris HJ. Evaluation of criteria for distinguishing atypical endometrial hyperplasia from well-differentiated carcinoma. Cancer. 1982;49(12):2547-59.

6. Liang S, Mu K, Wang Y, Zhang J, Sheng Y, Zhou J, et al. CyclinD1, a prominent prognostic marker for endometrial diseases. Diagn Pathol. 2013;8(1):1-8.

7. Lin L, Hicks D, Xu B, Sigel JE, Bergfeld WF, Montgomery E, et al. Expression profile and molecular genetic regulation of cyclin D1 expression in epithelioid sarcoma. Mod Pathol. 2005;18:705-9.
8. Hong A, Davies S, Stevens G, Lee CS. Cyclin D1 overexpression in AIDS related and classic Kaposi sarcoma. Appl Immunohistochem Mol Morphol. 2004;12:26-30.

9. Horvai AE, Kramer MJ, O’Donnell R. Beta-catenin nuclear expression correlates with cyclin D1 expression in primary and metastatic synovial sarcoma: a tissue microarray study. Arch Pathol Lab Med. 2006;130:792-8.

10. Kim JK, Diehl JA. Nuclear cyclin D1: an oncogenic driver in human cancer. J Cell Physiol. 2009;220:292-96.

11. Lancaster JM, Powell CB, Chen LM, Richardson DL. SGO Clinical practice committee. society of gynecologic oncology statement on risk assessment for inherited gynecologic cancer predispositions. Gynecol Oncol. 2015;136(1):3-7.

12. Sherman ME. Theories of endometrial carcinogenesis: a multidisciplinary approach. Mod Pathol. 2000;13:295-308.

13. Quddus MR, Latkovich P, Castellani WJ, Sung CJ, Steinhoff MM, Briggs RC, et al. Expression of cyclin D1 in normal, metaplastic, hyperplastic endometrium and endometrioid carcinoma suggests a role in endometrial carcinogenesis. Arch Pathol Lab Med. 2002;126:459-63.

14. Suri V, Shaffy, Sharma K. Expression of Cyclin D1 in normal, hyperplastic and neoplastic endometrium. Int J Recent Sci Res. 2017;8(5):17003-7.

15. Tsuda $H$, Yamamoto $K$, Inoue $T$, Uchiyama $I$, Umesaki N. The role of p16-cyclin D/CDK-pRb pathway in the tumourigenesis of endometrioid-type endometrial carcinoma. Br J Cancer. 2000;82:67582.

16. Chaudhary M, Bansal S. Expression of Cyclin D1 in endometrial hyperplasia and carcinoma. Ind J Pathol Microbiol. 2007;50:708-10.

17. Kala M, Gill M, Gupta V, Srivastava D, Tanwar P. Cyclin D1 expression in hyperplasia and carcinoma of the endometrium and its correlation with histologic grade and tumor type. J Gynecol Surg. 2014;30:1-4.

18. Nishimura Y, Watanabe J, Jobo T, Kato N, Fujisawa $\mathrm{T}$, Kamata $\mathrm{Y}$ et al. Cyclin D1 Expression in Endometrioid type endometrial adenocarcinoma is correlated with histological grade and proliferative activity, but not with prognosis. Anticancer Res. 2004;24:2185-92.

Cite this article as: Kundu PR, Sindhu A, Kaur S, Kulhria A, Hooda R. Expression of Cyclin D1 in normal and hyperplastic endometrium. Int J Reprod Contracept Obstet Gynecol 2019;8:4474-8. 